\title{
Introduction to the thematic issue
}

\author{
Juan A. Botía ${ }^{a,}{ }^{*}$, Simon Egerton ${ }^{b}$ and Hedda R. Schmidtke ${ }^{c}$ \\ ${ }^{a}$ Departamento de Ingeniería de la Información y las Comunicaciones, Universidad de Murcia, Spain \\ E-mail: juanbot@um.es \\ ${ }^{\mathrm{b}}$ School if IT, Monash University, Australia \\ E-mail: Simon.Egerton@monash.edu \\ ${ }^{\mathrm{c}}$ Carnegie Mellon University, Rwanda and USA \\ E-mail:schmidtke@cmu.edu
}

\section{Introduction}

This special issue is focussed on context awareness aspects within Smart Environments. Context awareness is one of the key features of Smart Environments, touching all layers of Ambient Intelligence from sensors and networks to Artificial Intelligence and interaction modalities. One of the questions related to Artificial Intelligence is, for example, how to appropriately represent context, or how to enable a smartphone to know a user's context and to adapt its behavior so as to optimally support them.

Appropriate handling of context information is central to enabling intelligence in the services and applications offered in smart environments. Intelligence is the key for adaptive systems and adaptiveness is the key for Smart Environments that support their inhabitants in a non-obtrusive manner that facilitates everyday activities. We believe that this long term usefulness will pave the way towards commercially successful Smart Environments.

Research about context regards several key parameters: among the most important, and best established is location. Location based services are among the first economically successful context aware systems, and location is still an active research topic within the Smart Environments field. However, context information also includes time, user interests and preferences, social context, current activity, plans and schedules. As systems shift from the labs of researchers into the homes of customers, cognitive aspects of the human user have to be considered, and concepts of personalization and long term user monitoring need to be ad-

*Corresponding author. dressed. The contributions to this Thematic Issue show context information as an enabler for advanced functionality in Smart Environment services.

\section{In this thematic issue}

The article "Towards interactive smart spaces" by Gilman et al. addresses aspects of social context as well as technologies necessary for interaction within and with smart spaces. The authors classify four types of interaction in a Smart Environment, and conclude outlining questions for a research agenda regarding how human social interaction can be facilitated by a Smart Environment.

Interaction modalities with a Smart Environment are another key question for context aware systems. Griol et al. address this question with an innovative example of how to enhance user interaction in "Providing personalized Internet services by means of contextaware spoken dialogue systems". The authors propose an enhanced dialogue based system which dynamically incorporates context when accessing a concrete service, where the parameters of context addressed are location, preferences and needs. The authors argue that spoken dialogue systems are becoming an important alternative to traditional graphical interfaces, and context-awareness clearly helps the system to generate natural and efficient dialogues.

Interaction modalities are also addressed in the article "A context-aware service provision for smart environments based on the user interaction modalities" by Gouin-Vallerand et al. The proposed system aims to use context information to select the right services for the right user at the right moment. The system is able to adjust its interaction to specific needs of a user, 
including the often neglected group of users with interaction challenges. The proposed adaptive technology makes it possible to deliver Smart Environments to a wide and diverse range of users.

The core technology of hierarchical location models is discussed in the article by Afyouni et al. titled "Context-Aware Modelling of Continuous LocationDependent Queries in Indoor Environments". The authors present a granularity-dependent query language and novel location modeling technique that can be used for navigation systems for indoor environments.

Context aware applications are discussed in the articles by Han et al. and Borrego-Jaraba et al. The article "Context-based Microblog Browsing for Mobile Users" by Han et al. presents an application that can help a user filter relevant Microblog entries depending on spatial and temporal context. The authors can thus distinguish between currently, locally relevant topics and topics of general interest. Moreover, they take into account user history, social relationships, and user ac- tivities to filter for those topics that could be relevant dependent on the user's interest.

A system that uses spatial and other context information to make educational resources in a university environment accessible is presented by Borrego-Jaraba et al. "An NFC based Context-aware Solution for Access to Bibliographic Sources in University Environments" shows an example of an application that uses technologies for context awareness such as NFC to enable the key parts of a concrete application.

\section{Acknowledgements}

We are grateful to the reviewers for their valuable contribution to this Thematic Issue. We would also like to thank the editorial staff, without whose support and guidance this effort would not have been possible. And finally, thanks to all the authors, whose contribution is the core of this Thematic Issue. 